\title{
Coemergence of Regularity and Complexity During Neural Network Development
}

\author{
E. Fuchs, ${ }^{1,2}$ A. Ayali, ${ }^{1}$ A. Robinson, ${ }^{1}$ E. Hulata, ${ }^{2}$ E. Ben-Jacob ${ }^{2}$ \\ ${ }^{1}$ Department of Zoology, Tel-Aviv University, Tel-Aviv 69978, Israel \\ ${ }^{2}$ School of Physics and Astronomy, Tel-Aviv University, Tel-Aviv 69978, Israel
}

Received 15 March 2007; accepted 21 June 2007

\begin{abstract}
With the growing recognition that rhythmic and oscillatory patterns are widespread in the brain and play important roles in all aspects of the function of our nervous system, there has been a resurgence of interest in neuronal synchronized bursting activity. Here, we were interested in understanding the development of synchronized bursts as information-bearing neuronal activity patterns. For that, we have monitored the morphological organization and spontaneous activity of neuronal networks cultured on multielectrode-arrays during their self-executed evolvement from a mixture of dissociated cells into an active network. Complex collective network electrical activity evolved from sporadic firing patterns of the single neurons. On the system (network) level, the activity was marked by bursting events with interneuronal synchronization and nonarbitrary temporal ordering. We quantified these individual-to-collective activity transitions using newly-developed system level quantitative
\end{abstract}

measures of time series regularity and complexity. We found that individual neuronal activity before synchronization was characterized by high regularity and low complexity. During neuronal wiring, there was a transient period of reorganization marked by low regularity, which then leads to coemergence of elevated regularity and functional (nonstochastic) complexity. We further investigated the morphology-activity interplay by modeling artificial neuronal networks with different topological organizations and connectivity schemes. The simulations support our experimental results by showing increased levels of complexity of neuronal activity patterns when neurons are wired up and organized in clusters (similar to mature real networks), as well as network-level activity regulation once collective activity forms. (C) 2007 Wiley Periodicals, Inc. Develop Neurobiol 00: 000-000, 2007

Keywords: functional complexity; neuronal network; in vitro; development; spontaneous activity

\section{INTRODUCTION}

The dynamics of evolved connectivity and functionality during neuronal systems development is a subject of major interest. There are many examples in which throughout the organizational process of neuronal assemblies, neurons discharge endogenous spontaneous bioelectrical activity (Zhang and Poo,

Correspondence to: A. Ayali (ayali@post.tau.ac.il) or E. BenJacob (eshelbj@gmail.com).

Contract grant sponsors: National Institute for Psychobiology in Israel, Israel Science Foundation (ISF), Tauber Fund.

(c) 2007 Wiley Periodicals, Inc.

Published online in Wiley InterScience(www.interscience. wiley.com). DOI 10.1002/dneu.20557
2001). The early activity patterns were suggested to play a central regulatory role in the formation of functional neuronal circuits (Katz and Shatz, 1996; Ben-Ari, 2001; Zhang and Poo, 2001; Corner et al., 2002; Hua and Smith, 2004; Marder and Rehm, 2005). In addition to well-studied "small-scale" activity-dependent processes, in which activity is tightly correlated in time and space [e.g., neurite growth and retraction (Cline, 2001; Wong and Ghosh, 2002) or synaptic stabilization (Hua and Smith, 2004)], broader scale temporal and spatial correlations, at the macroscopic level, were also suggested to encode important information for guiding circuit development (Feller, 1999). A fundamental, macroscopic level, activity pattern shared by many different developing 
neuronal systems is that of spontaneous rhythmic bursts of action potentials. These are correlated across many cells and occur with a periodicity on the order of minutes (Feller, 1999). Examples can be found in many developing systems that are characterized by increased interneuronal connectivity and high overall level of activity, both, in vivo as well as in vitro (O’Donovan and Chub, 1997; O’Donovan, 1999; Streit, et al., 2001).

Self-assembled in vitro neuronal networks are widely used as an experimental model system for studying the basic mechanisms governing neuronal networks activity; their collective dynamical modes, information processing, and learning capabilities (Habets et al., 1987; Maeda et al., 1995; Kamioka et al., 1996; Marom and Shahaf, 2002; Opitz et al., 2002; Segev et al., 2002, 2004; Baruchi and Ben-Jacob, 2007). In these reports, cultured neuronal networks evolve from dissociated cortical or hippocampal neurons and glia spread homogeneously over suitable substrates. The cells regenerate neuronal processes (axons and dendrites) and consequently self-assemble into elaborate neuronal networks. During network maturation, neurons spontaneously discharge repetitive multiunit bursts of action potentials (Maeda et al., 1995; Potter, 2001; Segev et al., 2002; Tateno et al., 2002). The degree of synchronization between firing units increases as the networks develop (Habets et al., 1987; Kamioka et al., 1996; Tateno et al., 2002; Van Pelt et al., 2004; Chiappalone et al., 2006; Wagenaar et al., 2006b). In mature networks, the spontaneous activity is marked by synchronized burst events (SBEs)-short time windows (hundreds of milliseconds), during which most of the recorded neurons participate in relatively rapid firing separated by long (seconds) intervals of sporadic neuronal firing.

From a dynamical systems perspective, the SBEs are collective modes of activity, each with its own specific complex spatio-temporal pattern of neuronal firing. They exhibit nonarbitrary temporal ordering characterized by scale-free statistics and long-range correlations. These features, which have been identified in cultured networks composed of mammalian cortical neurons (Segev et al., 2002, 2004; Hulata et al., 2004; Raichman et al., 2006; Wagenaar et al., 2006a; Baruchi and Ben Jacob, 2007), were recently also described in networks obtained from a relatively simple invertebrate motor ganglion-the locust frontal ganglion (Ayali et al., 2004).

Use of the relatively large locust neurons, which can be cultured in low densities, previously enabled us to provide a continuous and detailed description of the neuronal networks' morphological organization through its distinct developmental stages (Shefi et al., 2002a, 2005). Furthermore, comparative investigation of a distinct neuronal population operating under different levels of structural and functional constraints have also been conducted (Ayali et al., 2004). The temporal organization of neuronal activity was characterized using an analytical method developed to characterize the dynamic activity of biological time series (Hulata et al., 2004, 2005; Fuchs et al., 2006; Ayali et al., 2007). By extracting maximal information about the sequence's temporal organization, this analysis provided an estimate of the sequence regularity as well as its potential maximal capacity to carry information to be utilized for information processing tasks (functional complexity). As suggested by Ben Jacob and Levine (2001), this quantification is based on the discrimination between autonomous biotic complexity (generated via autonomous utilization of internally-stored means and thus self-regulated) and nonautonomous, abiotic complexity.

In the present study, we utilized simultaneous multisite continuous recording of electrical activity of individual locust ganglion neurons in combination with our recently developed system level analyses methods. We systematically quantified the recorded activity throughout neuronal development from the nonwiring stages to the highly connected mature networks in terms of its regularity, structural, and functional complexity. We further tested the observed features of network structural and functional development using simulations of artificially modeled networks. We provide a novel description of the spontaneous emergence of the networks system-level activity patterns and discuss our results in relation to fundamentals of the very widespread phenomenon of neuronal oscillation and synchronization.

\section{METHODS}

\section{Cell Culture and Activity Recording}

The procedures for dissection and dissociation of locust frontal ganglion (FG) neurons for primary cell culture have been previously described in detail (Shefi et al., 2002a). Briefly, following dissection, neurons were dispersed by enzymatic treatment and mechanical dissociation. The neurons were plated on planar multi electrode arrays (MEA, Multi-Channel Systems), precoated with a mixture of Concanavalin A Type IV and poly-D-lysine. Culture media (L15, Sigma) was enriched with 5\% locust hemolymph and changed once a week. Plated cell cultures were placed on the MEA board (B-MEA-1060, Multi-Channel Systems) for simultaneous long-term noninvasive recordings of neuronal activity from several neurons at a time. Throughout, the cells were maintained in darkness, under controlled temper- 
ature and humidity. Recorded signals were digitized and stored for off-line analysis on a PC via an A-D board (Microstar DAP) and data acquisition software (Alpha-Map, Alpha Omega Engineering). Spike sorting of the extracellular recordings was performed by an algorithm based on wavelet packets decomposition (Hulata et al., 2000), to identify single and distinctive units. Analysis was based on optical observations of single cells and network morphology and on neuronal activity recorded from 10 cultured preparations over the course of 2 weeks in vitro. Overall, between 1 and 9 neurons were successfully recorded and analyzed from each of the different preparations.

\section{Representations of the Recorded Activity}

Recorded signals were transformed into ordinary binary time sequences (with $10 \mathrm{~ms}$ time bins) whose "1"s mark detected action potentials. Next, for each activity sequence manifested by burst activity, we evaluated its corresponding sequence of neuronal burst events. The burst time positions were determined by searching for high (10-fold higher than baseline activity) local maxima in spike density function (convoluted with a smooth kernel function), and then represented in a binary sequence of renormalized time intervals between successive events (each bin width, $\tau$, was the typical time width of the event, around $1 \mathrm{~s}$, thus both spikes and bursts were described as point processes).

\section{Temporal Structure Complexity and Regularity of Recorded Sequences}

Neuronal information is encoded both in activity rates and their relative temporal location. Signals of a complex temporal nature, characterized by local and global variations, can demonstrate large frequency variations at each temporal location, when looking at time windows of different widths. These local variations will also vary from time point to time point along the sequence. The algorithm for analyzing signal regularity and complexity has been fully described elsewhere (Hulata et al., 2004, 2005), and is based on the calculation of homogeneity and variability of a sequence's optimal representation in its corresponding time-frequency domain, which enables a compact representation of both local and global variations. An example of such time-frequency representation is given in Figure 1. The domain was tiled (partitioned) into $N$ rectangles, each with its own height $\Delta f$, and width $\Delta t$ representing the relative "energy" $\left(q_{n}\right)$ of the frequency range $\Delta f$ in the time window $\Delta t$, using wavelet packet decomposition in a way that extract maximal information on the sequence temporal ordering (maximizing the global information measure $M$ $\equiv-\sum q_{n} \log q_{n}$ ). The sequence regularity (RM) is a measure of the uniformity of the constructed time-frequency plane by calculating the mean similarity of tile proportions as follows: the relative resolution of each rectangle $n$ is $R_{n}=\frac{\log _{2}(\Delta t / \Delta f)}{\log _{2} N} ;-1 \leq R_{n} \leq 1$ and the regularity measure $(\mathrm{RM})$ is defined as the average of $R_{n}: \mathrm{RM}=\frac{1}{N} \sum_{n=1}^{N} R_{n}$.

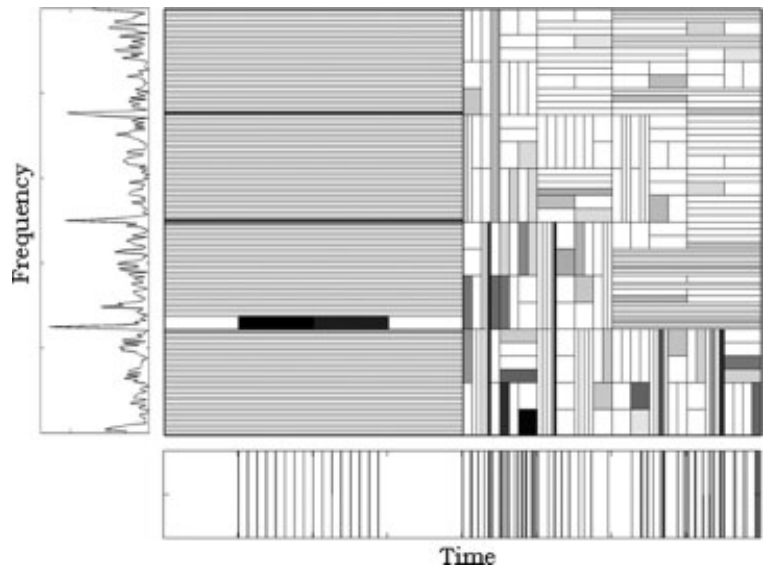

Figure 1 Best Tiling representation. At the bottom, we present a binary signal, which is composed of two types of signals: a periodic sequence (at the left) and a sequence whose intervals were drawn from a Levy-type distribution function (at the right). On the left panel, the power spectrum plot shows the global signal frequency contents. A wavelet-packet-decomposition algorithm allowed partitioning of the time-frequency plane into rectangles that can capture most efficiently the information about both local and global variations in the sequence. The color of each rectangle (from light to dark) in the tiling map represents the portion of the specific frequency interval in the corresponding time location. As can be clearly seen, the periodic sequence shows similar features throughout the sequence length in straight forward manner around the segment's spectral density. In contrast, the segment with a Levy-type intervals distribution is characterized by local temporal variations and therefore its features cannot be well-captured and demonstrated using global power-spectrum analysis. The timefrequency domain of this multifarious signal is characterized by different rectangles with varied proportions.

The regularity measure (RM) estimates the content of periodic phenomena within the sequences of recorded activity. A purely disordered, randomly distributed sequence will result in a value of 0 , whereas a purely periodic sequence (Fig. 1, left) will result in a value of 1 . In between pure order and disorder, the content of periodicity in a biological signals will include noisy periods, local periods changing over the coarse of time or multiple frequencies expressed simultaneously (Fig. 1, right).

A complementary observable- the structure complexity of a sequence-is associated with the plane local (intrasegment) and global (intersegment) variability. Intuitively, one would recognize that both the purely ordered and the disordered sequences have little variation in their temporal structure. A randomly distributed sequence may have some stochastic local variations, but no global variation as long as the probability function does not vary. On the other hand, a purely periodic sequence (Fig. 1, left) has no variation at all. In our biological recordings, profound temporal structure is related to the existence of bursting events, of 
long-tail distributions (Fig. 1, right) or evolving probability functions (Segev et al., 2002; Ayali et al., 2004).

The local variability is calculated in short segments of the entire sequences (named words). For each word 1, we calculate the amount of nonhomogeneity of the tiling (VF)

$$
\mathrm{VF}_{l}=\left(\frac{N_{E}(l)-\overline{N_{E}}}{\overline{N_{E}}}\right) \frac{\sum_{n, m}\left|R_{n}-R_{m}\right| \cdot \Theta\left(q_{n} \cdot q_{m}\right)}{\sum_{n, m} \Theta\left(q_{n} \cdot q_{m}\right)}
$$

where the sum is the overall neighboring rectangles $n$ and $m$. $N_{E}(l)$ is the number of events detected within the $l$-th word, and $\overline{N_{E}}$ is an average over different words of the same length as the $l$-th one. $\Theta(x)$ is the heavyside function; $\Theta(0)=0$ and $\Theta(x \neq 0)=1$. The global variability is quantified by the variation of these local word values. For a sequence segmented into $N_{\mathrm{w}}$ words, we calculate the structural complexity (SC) observable to be:

$$
\mathrm{SC} \equiv \operatorname{var}(\mathrm{VF}) \equiv \frac{1}{N_{w}} \sum_{l=1}^{N_{w}}\left(\mathrm{VF}_{l}-\overline{\mathrm{VF}}\right)^{2}
$$

We select the length of the word to be the one that maximizes this global variation, so that the time scale of the effective analysis results from the sequence characteristics and not arbitrarily selected.

Throughout, we used both the SC and RM observables for signal analysis. To describe the change in SC and RM values of recorded traces along time, we segmented the sequences into partially overlapping 1-h long segments and applied the analysis on each segment separately.

\section{From Temporal Structure Complexity to Functional Complexity}

The temporal structure of a recorded sequence is composed of several motifs. At a first glance, the sequence is a realization of a probability distribution function. Since this distribution function of the interevent-intervals usually has longtail decay (Segev et al., 2002; Ayali et al., 2004; shown also in this article), there is a basic stochastic temporal structure of the intervals. The relation of our measures and this distribution function has been explored in previous studies (Hulata et al., 2004, 2005). We have shown there that for neuronal recordings, the mean values of $\mathrm{RM}$ and $\mathrm{SC}$ for many shuffled sequences resemble those of artificially constructed sequences with similar distribution function parameters. Furthermore, in such analysis, it is recognized that biological recordings may have more temporal structure than this basic stochastic content. This quantity can be measured by calculating the difference between the $\mathrm{SC}$ of a recorded sequence and the average SC of different randomly shuffled sets of the sequence intervals (sequences after shuffling have exactly the same interval distribution as of the original one but in random temporal ordering). We normalize the difference by the total amount of SC to receive the fraction of complexity lost by shuffling which indicate on the amount of information encoded in the specific temporal ordering. We named this fraction "the functional complexity": $\mathrm{FC}=(\mathrm{SC}-\mathrm{SCs}) / \mathrm{SC}$, where SCs is the mean SC value of the sequence's random permutations. In a sense, one can view the basic-stochastic structure of recorded activity sequences as being the "infra-structure" in which a network operates, defining the basic time-scales and rate dynamic ranges in which the network can fire. The functional complexity serve as a measure of how much of this "infrastructure" is practically used, thus creating profound temporalstructures that are correlated in time and as a result are lost when the sequence is shuffled.

\section{Simulations-Modeling Neuronal Networks with Different Topological Organizations and Connectivity Schemes}

Adopting the generic dynamical model designed by Volman et al. (2004), we modeled artificial neuronal networks with different connectivity configurations. In these model networks, both neurons and the synapses connecting them were described as dynamical elements: the neurons were modelled by the Morris-Lecar dynamical description (Morris and Lecar, 1981) exchanging action potentials via the multistate dynamic synapses, as described by Tsodyks et al. (2000). All modelled networks were composed of 100 cells (30\% of the cells were set as inhibitory) forming connections according to either nearest neighbour connectivity rule [neurons $(i, j)$ are connected in probability $p_{1}$ if the distance between them is smaller than a chosen threshold $d(i, j)<D$ and not connected otherwise], or in clustered topology in which the neurons are organized in 10 clusters of 10 cells each, so that the probability for connections between two neurons within a cluster, $p_{\text {in }}$, was set to be 20 times higher than $p_{\text {out }}$ - the probability to form connections between neurons of different clusters. For both topological organizations, we simulated networks with different overall levels of connectivity while keeping the connectivity rule set-increasing the values of $p_{1}, p_{\text {in }}, p_{\text {out }}$ while keeping $D$ and the ratio $p_{\text {in }} / p_{\text {out }}$ constant. We ran the simulations for $500 \mathrm{~s}$ under each condition and connectivity rule. Activity sequences had then been analyzed in a similar way to that employed for the experimental data (cultured networks).

\section{RESULTS}

\section{General Description of the Observed Activity of Cultured Neurons}

Dissociated neurons from the locust FG were successfully cultured on MEA surfaces, allowing prolonged simultaneous recordings from several neurons as cell bodies of the plated neurons regenerated neural processes, connected to neighboring cells, and throughout the network maturation. Spontaneous activity of single neurons was observed starting at 4 days in vitro, when only primary connections between adjacent 
A.

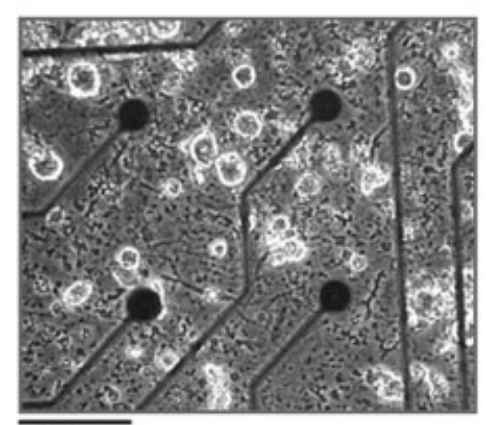

B.

$$
100 \mu \mathrm{m}
$$
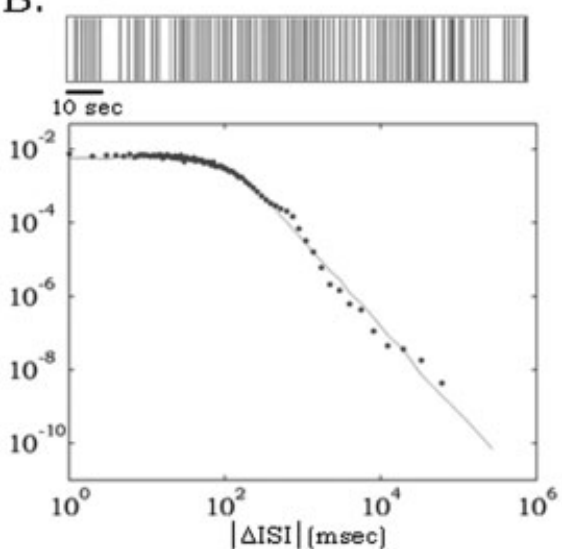

C.

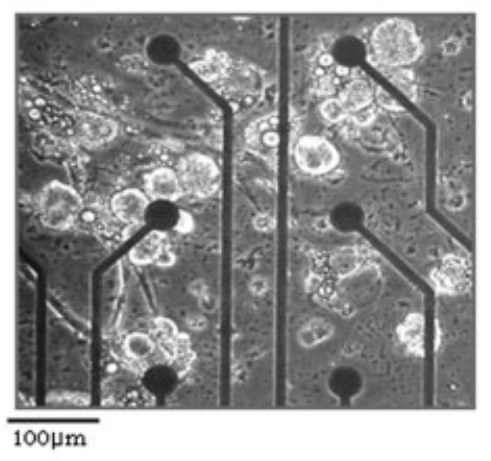

D.

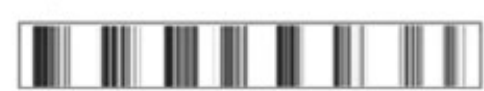

$20 \mathrm{sec}$

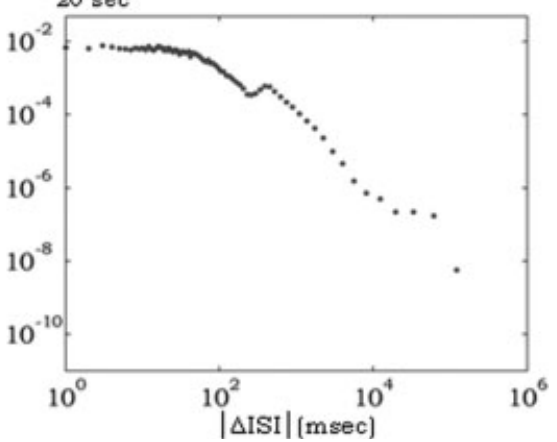

Figure 2 Structural organization and spontaneous electrical activity of FG neurons cultured on a multielectrode array during the first week in vitro. The structural organization of cultured neurons 4 (A) and 5 (C) days after plating shows neuronal organization into small cell clusters. (B) Sporadic spontaneous firing of a single neuron 4 days after plating in a raster plot representation (upper trace). The probability density function of its spike interval increments (absolute values) is presented on a log-log scale. Solid line is a fitted levy curve (D) The firing pattern

cells could be observed [Fig. 2(A,B)]. All neuronal activity sequences recorded at $4-5$ days after plating were characterized by sporadic firing of action potentials. Averaged frequencies were in the range of 1-10 $\mathrm{Hz}$ [a raster plot example is shown in the upper panel of Fig. 2(B)]. By the end of the first week, both the networks' structural organization and the cells' firing patterns had undergone major changes. A marked decrease in the rapid initial neurite growth rate was observed and the cell bodies started to migrate, aggregating into packed clusters [Fig. 1(C), in accordance with Shefi et al., 2002a; 2005]. A pronounced change in the corresponding neural firing pattern accompanied this morphological rearrangement: quiescent periods appeared and the pattern of sporadic firing changed into alternations between active and nonactive periods [Fig. 2(D)]. This transition was observed in all cases in which prolonged continuous recordings were obtained from a single cell during this developmental stage $(n=4)$, and only during this stage.
We computed sequences of interval increments (a sequence of the difference between sequential interspike-intervals) and plotted their distribution on a $\log -\log$ scale for dynamic scaling characterization and representation of firing rate variations. The probability density function of the increments could be approximated by a Lévy distribution (a generalization of the Gaussian distribution for processes with diverging variance) over 4-5 decades [Fig. 2(B,D)]. The changes in firing pattern were reflected by elevated probabilities of specific firing rates within bursts and between bursts [compare Fig. 2(B,D)].

\section{Increased Temporal Structure Complexity During Network Development-Burst Appearance}

Temporal ordering of neuronal activity provides a template for information coding. We utilized the tools 
A.

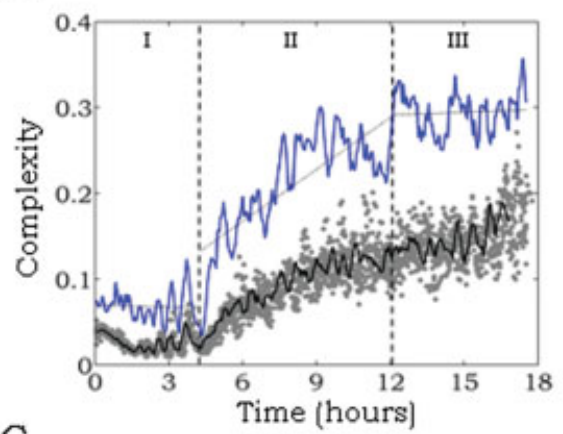

C.

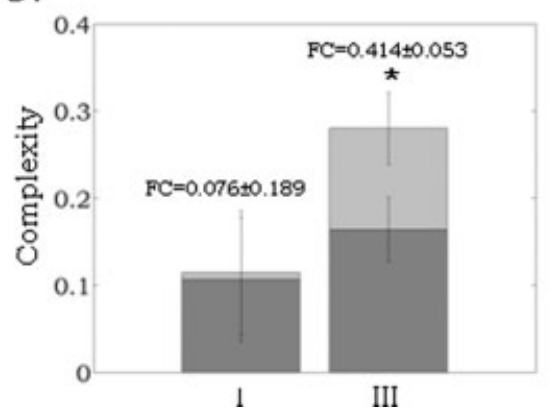

B.

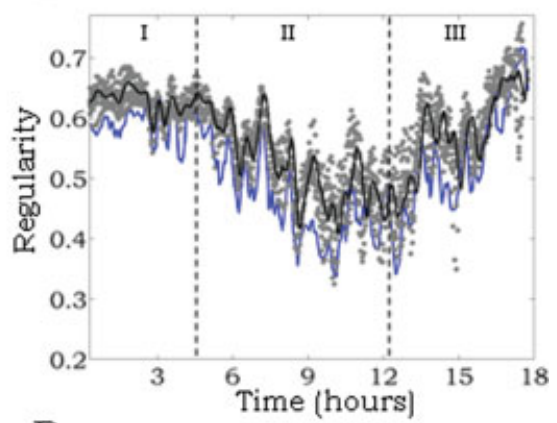

D.

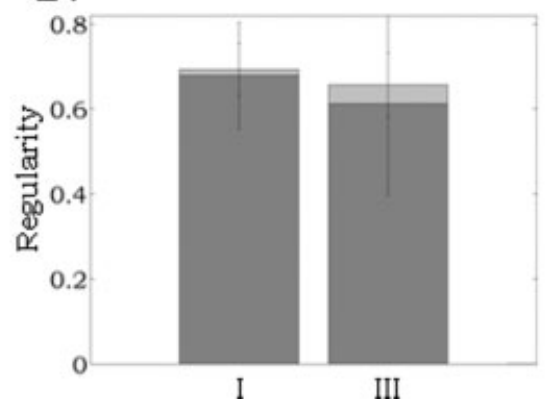

Figure 3 A continuous description of structural complexity (A) and the corresponding regularity values (B) of the activity sequences recorded between the two time points shown in Figure 1. Blue lines describe the complexity and regularity values calculated for the experimental data. The gray dots represent values obtained after random shuffling of the original sequences; each point corresponds to the result of one of five different shuffling procedures. The development of a burst temporal organization is reflected by a marked increase in sequence complexity. A region of low $(<0.5)$ regularity values was obtained during the transition between the two stable states (spiking and bursting). Three regression models that best describe the change dynamics are plotted in grey lines and the zone limits of each regression model are marked (I, II, and III). (C) and (D) show the mean $( \pm$ std dev) values of recorded traces before and after transition to burst firing (corresponding to Zones I and III in the example presented in panel A, $n=4)$. A significant increase $(p<0.01)$ in the complexity of the firing pattern is seen, with no change in the regularity values. The dark gray area of each bar represents the mean value obtained after random shuffling of the sequences. The light area of the bars represents the functional complexity (see text). This was found to be significant only after transition to burst firing ( $p<0.05$ paired $t$-test).

of structural complexity versus regularity to study the evolvement of neuronal activity patterns throughout the process of network organization. As mentioned earlier, the complexity algorithm was developed to estimate the potential maximal "information capacity" of recorded sequences and to identify selfregulation motives in the observed activity patterns. An example of the dynamics of the values calculated from neuronal recordings during the transition to a burst firing pattern, as well as averaged data, is given in Figure 3. Data in Figure 3(A,B) were calculated from an 18-h continuous recording of a single neuron, starting from an activity pattern similar to that presented in Figure 2(B) $(t=0$ in the figure -4 days after plating) until it changed to that shown in Figure 2(D). A clear transition can be seen between two rela- tively stable states/phases: low structural complexity values characterizing the initial phase of spiking activity [zone I in Fig. 3(A)] and high values calculated after burst formation (Zone III). In both zones, sequence regularity is relatively high, reflecting their nonrandom and stationary nature (both their frequency content and their statistical parameters are constant over time). However, within the process of firing mode change (Zone II), the signal's nonstationariness is reflected in its decreased regularity, which retains its high level only after the transition into bursts firing. The averaged data demonstrate the significant increase in the structural complexity of the activity patterns during this developmental stage [Fig. 2(C)], whereas the regularity retained its previous level [Fig. 2(D)]. 
A.

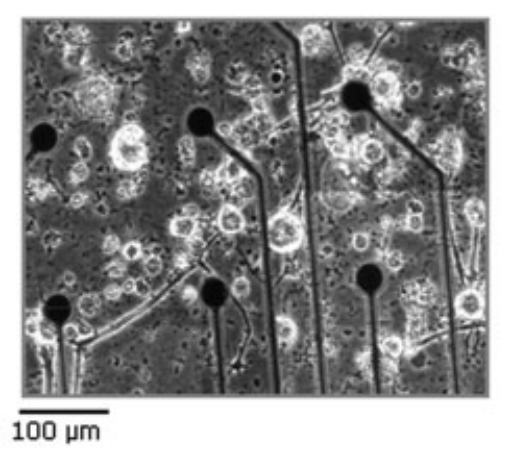

B.
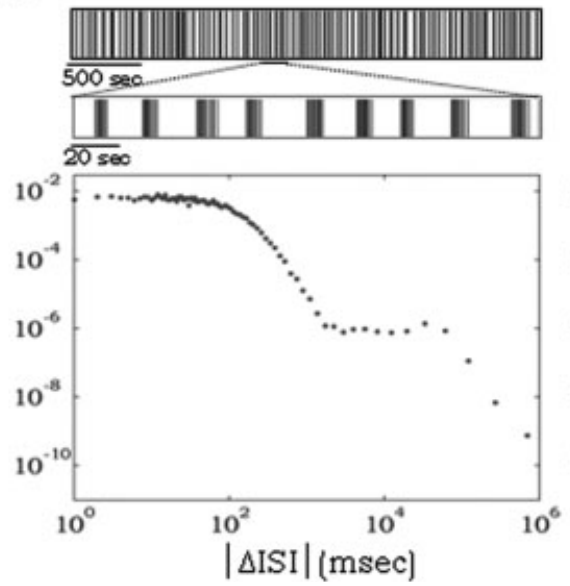

C.

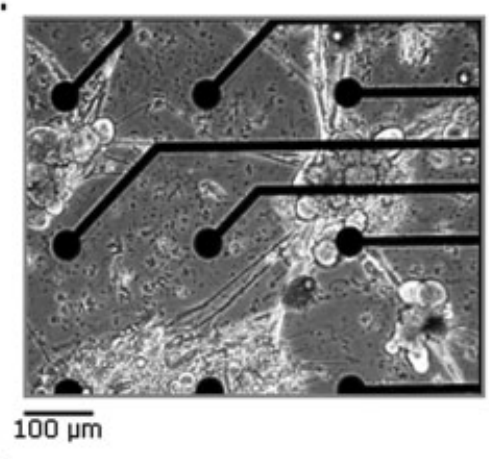

D.
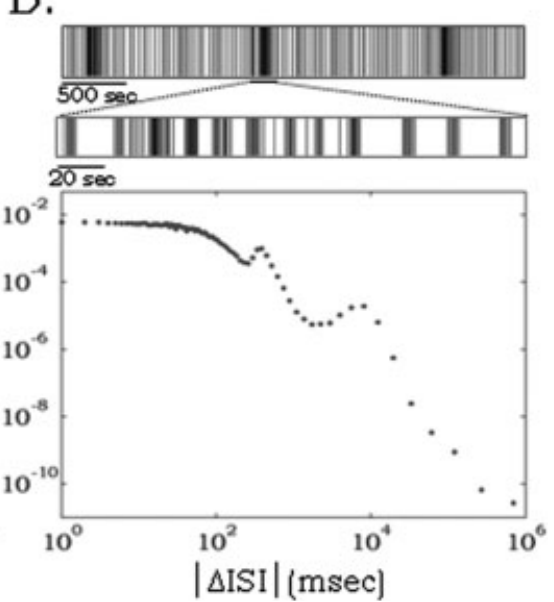

Figure 4 Structural organization of cultures and temporal organization of spontaneous electrical activity during the beginning $(\mathrm{A}, \mathrm{B})$ and the end $(\mathrm{C}, \mathrm{D})$ of the second week after plating. Recorded sequences (upper panels in B and D) are shown in two different time scales (the lower ones are enlargements of areas marked with bars in the upper ones) to demonstrate the change in the temporal order of bursts. The appearance of "bursts of bursts" is demonstrated in the event distribu-

\section{From Temporal Structure Complexity to Functional Complexity}

A comparison of the values calculated for recorded versus shuffled sequences allowed us to identify the amount of information encoded in the specific temporal ordering, beyond their basic stochastic content. As can be seen in Figure 3, the elimination of temporal ordering (and hence causality) resulted in a decrease in the complexity. We referred to the difference between the values calculated for the original and shuffled sequences as the "functional complexity." Functional complexity thus measures the content of nonstochastic temporal structure in a time series; hence, as this value grows, so does the information capacity of the sequence.

The comparison of functional complexity of recorded sequences along the network development demonstrates that the increase in complexity of activity patterns during development does not merely result from changes in interval distribution, but also from the complexity of their precise temporal ordering.

\section{Activity Dynamics During Later Stages of Network Development}

Once formed, bursts represented the dominant pattern of network activity within all recorded preparations. Their inner structure, temporal ordering, and synchrony within the network changed along the network development. An example of a network structural organization and its corresponding activity patterns in later stages of development is shown in Figures 4 and 5. Cell clusters increased in size, aggregating ensembles of small clusters, connected to other distant cell clusters by thick nerve-like bundles. Synchronous activity between several neurons appeared after 10 days in vitro. An example of this rich, partially synchronized 

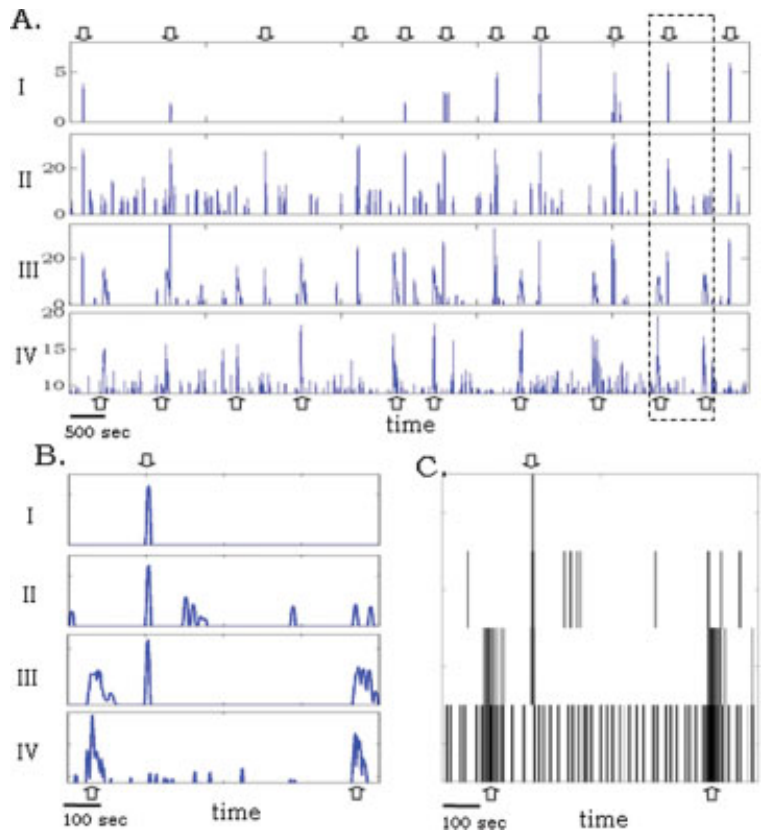

Figure 5 (A) Activity density functions calculated for four simultaneously recorded neurons from a mature culture (neuron I-IV, 15 days in vitro). The number of spikes in each time bin is plotted against time. Synchronized activity between channels is indicated by arrows. Arrows at the top refer to synchronized burst events in the three upper neurons, while the arrows at the bottom indicate a different burst type co-occurring in the third and fourth neurons. (B) An enlargement of the area marked in dashed bar shows two types of synchronized activity patterns. [Color figure can be viewed in the online issue, which is available at www.interscience.wiley.com.]

activity is shown in Figure 5. Spike density functions, calculated for each neuron separately, revealed intricate interneuronal correlations. Periods of higher density of activity occurred simultaneously in the first top three neurons. Such periods were not correlated with any activity changes in the fourth neuron. The latter (whose activity is presented in Fig. 3) burst more frequently and showed firing patterns organized in two hierarchical levels: bursts of action potentials (first organization level) were also organized in burst-like structures (second level). Thus, there were relatively short periods of intense burst discharge, separated by longer periods of lower frequencies of activity. Each burst of bursts in this neuron (neuron IV in Fig. 4; an activity threshold was used to present only the second hierarchical organization level) was correlated with a prolonged burst in neuron III. The latter demonstrated two firing patterns that coexist for many hours, each synchronized with a different functional group in the network.

\section{Temporal Structure Complexity Evolvement During the Later Stages of Network Development}

Temporal characteristics of burst sequences during development were evaluated by means of continuous description of their structural complexity and regularity (Fig. 5). Burst locations were detected and sequences of burst ordering were constructed similarly to the described analysis of spikes. Thus, analysis of burst sequences ignored spike organization within burst and revealed features concerning the bursts ordering.

The data shown in Figure 6 were obtained from burst sequences of relatively young (5-9 days in vitro, shortly after burst formation, $n=7$ ) and mature networks (10-15 days, $n=10)$. The significant increase in the structural complexity of the burst activity patterns during the network development was
A.

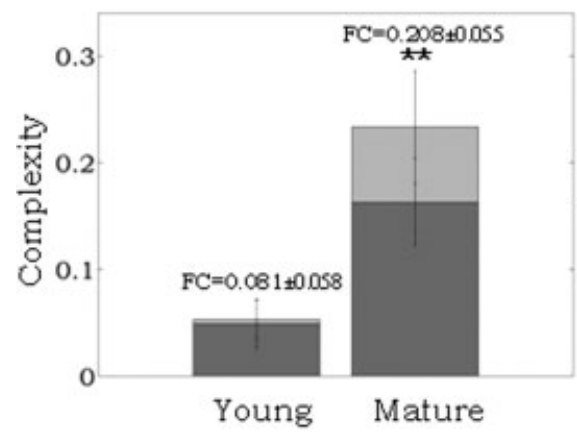

B.

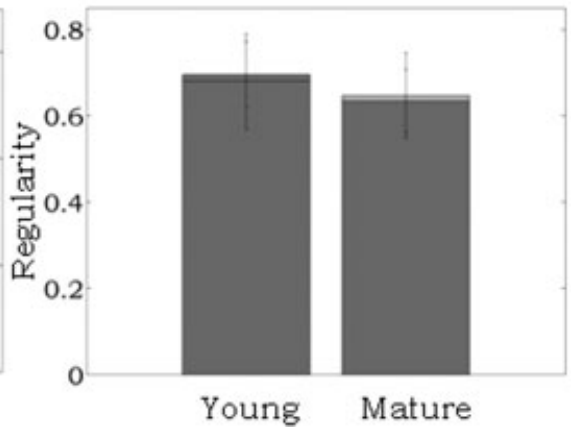

Figure 6 Mean ( \pm SD) structural complexity (A) and regularity (B) calculated for burst sequences recorded from neurons of young (5-9 days in vitro, shortly after first cluster formation, $n=7$ ), and mature networks (10-15 days in vitro, $n=10)$. A significant increase in activity pattern complexity is apparent, with no change in their regularity. Similar to the analysis of spike sequences in Figure 2, the change in burst sequence organization is characterized a by significant functional complexity in mature network patterns. 
A.
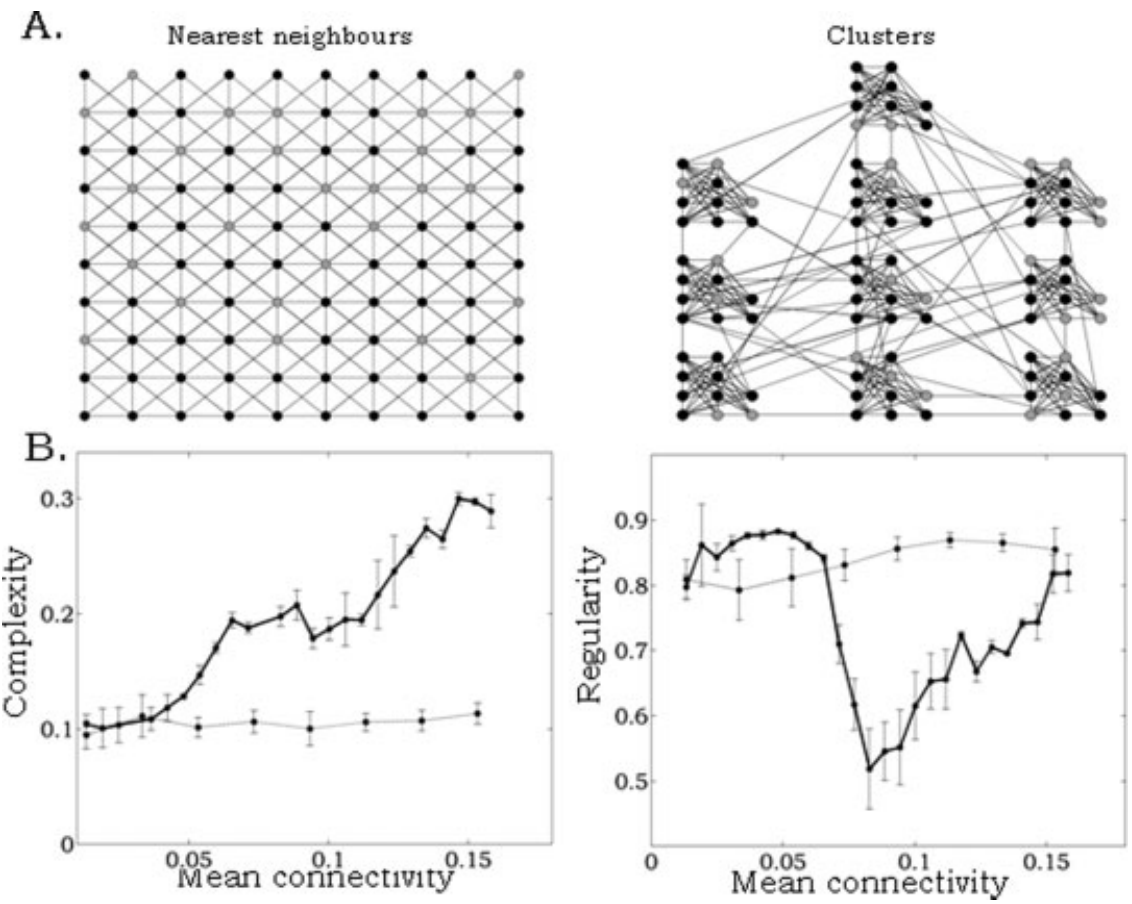

Figure 7 Modeling network's development. (A) Artificial networks with two basic topological organizations were simulated (gray and black dots correspond to inhibitory and excitatory neurons respectively). Increasing levels of connectivity mimicked the network development. (B) Complexity and regularity values were calculated for the firing patterns of each of the different networks throughout their development. Plotted values correspond to the mean ( \pm std dev) of all neurons in each network. Differences between the two network topologies (i.e., higher information capacities expressed by the clustered topology) revealed the importance of inhomogeneous spread of connections, a key feature of our real networks. Similar to our cultured neurons, the observed dynamics of activity patterns of the modeled network were characterized by increasing levels of complexity and transition to network-level regulation during network development.

apparent in all tested burst sequences and was not accompanied by a change in sequence regularity, which retained its previous level. Activity sequences of young networks were characterized by relatively periodic occurrences of bursts and therefore remained relatively similar upon intervals shuffling [Figs. 3(B) and 5]. In contrast, a significant functional complexity was obtained upon shuffling the sequences recorded from mature networks [Figs. 3(D) and 5], indicating that the highly complex firing patterns of mature networks were not arbitrary but rather composed of specific internal organizations of regulated activity patterns.

\section{Networks Modeling}

The activity of artificial neural networks with different topological organizations and connectivity levels (mimicking different developmental stages of our experimental networks) was analyzed to investigate changes in activity patterns during the process of net- work development. In these simulations, all neuronal properties, except for the connectivity pattern, were kept constant [the basic topological configurations are shown in Fig. 7(A)]. We simulated network's development (wiring up-the process of interneuronal connections) by gradually increasing connectivity levels of the modelled network, following either nearest-neighbor connectivity rules or clustered topology. In both topological configurations, as connectivity level increased neurons started to fire in a synchronous manner, sharing salient features of the cultured networks activity patterns. That is, in both configurations, the network-level operational mode was characterized by spontaneously generating multineuronal synchronized bursting events separated by long periods of sporadic activity. However, the analysis of firing patterns at the network-level operational mode revealed clear differences in the amount of information encoded by networks with different topological configurations. Figure 7(B) summarizes the amount of complexity and regularity encoded by the different 


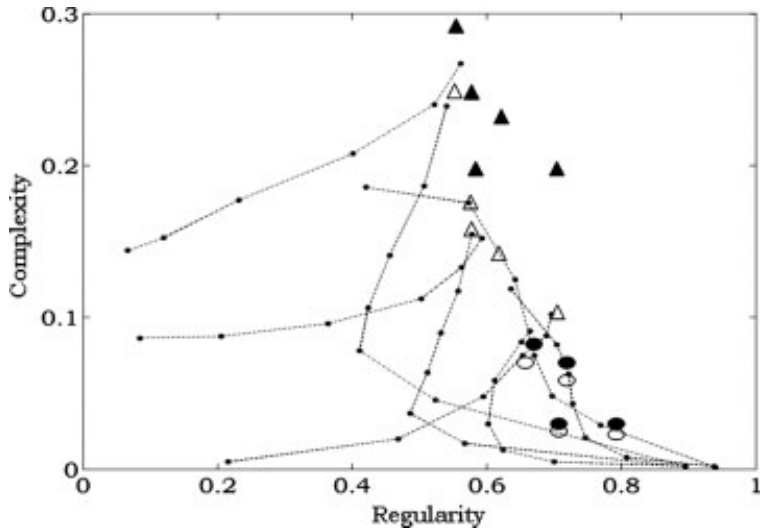

Figure 8 The values constructing the dotted lines were obtained from families of artificially constructed sequences with predefined statistical parameters used to explore the complexity-regularity plane. The characteristic maps demonstrate that elevated complexity values are obtained in the range of middle regularity values (curves are steepest in the central area). Several examples of recorded sequences (filled symbols) with statistical properties that match the artificially constructed sequences, as well as the corresponding shuffled sequences (open symbols) were plotted onto the plane according to their calculated complexity and regularity values. Representative data of both young and mature networks are shown (circles and triangles, respectively). Data demonstrate that the process of network development can be described as moving up along different branches of the characteristic map toward higher complexity values. Real data are always on the upper edge of the region of the corresponding shuffled results (shown as averages).

simulated networks. As can be seen, the organization of the networks into functionally coherent and sparsely interconnected clusters support high levels of activity patterns' complexity as well as networklevel activity regulation once collective activity forms. The transition to network level organization that is characterized by highly regulated and persistent synchronized bursting events was accompanied by temporary low regularity values, after which moderately high regularity levels were retained [Fig. 7(B)].

\section{Mapping the Complexity-Regularity Plane with Test Sequences}

Families of artificially-constructed signals with predefined statistical properties were used to explore the complexity-regularity plane to further understand the values obtained for the recorded sequences (Fig. 8). Clearly, both completely regular (periodic sequence) and purely random signals are not complex (they demonstrate low structural complexity values). The intermediate region between these extremities was analyzed by calculating the structural complexity and regularity values for various sequences artificially constructed to span the range between ordered and disordered signals. Each sequence was constructed by drawing a set of numbers out of a Lévy distribution with predefined parameters and considered as a realization of the distribution [more details can be found in (Hulata et al., 2004)]. For each set of parameters, we constructed many realizations, calculated the complexity and regularity values for each, and used the averaged values to construct the test-sequence characteristic-map in the complexity-regularity plane (dotted lines in Fig. 8 represent the averaged values of each family of constructed sequences). Each family of sequences is composed of one branch on the regular side (regularity $>0.5$, starting from purely periodic signals with narrow distributions and increasing their variability) and a second branch on the random side (regularity $<0.5$, starting from normal distributions). The branches meet at the boundary between the random and regular sides, in the area of relatively higher complexity. This region is where all our experimental results were mapped to (Fig. 6), i.e., the process of network development can be described as moving up along branches in this region of the map toward higher complexity values. As can be seen, the average values of our shuffled sequences approach the value calculated from artificially constructed sequences with the same statistical properties (i.e., data from shuffled sequences practically fall on the curves). The real data from our recorded sequences always have higher complexity. Hence, they will always be located at the outskirts of the distribution, at the extrapolated higher end of the corresponding curve (that was constructed with similar statistical properties).

\section{DISCUSSION}

The function of neuronal networks, from motor control to information processing, relies on the ability of the individual neurons to generate temporal sequences of action potentials and on modulation of the level and patterns of activity at the network level. Firing profiles of the neurons are synchronized to generate the collective dynamics of the network as a whole. Ubiquitous in different brain regions and phylogenetically preserved, synchronous network oscillations of several orders of frequency magnitude and network size have been shown to be crucial for brain functionality (Buzsaki and Draguhn, 2004). One of the great challenges is to understand the evolvement of the synchronous network activity during network development. 
We studied the development of in vitro networks of locust neurons as a model system for development, as well as function-form interactions, in nervous systems. Our approach was based on the assumption that fundamental principles would probably be more conspicuous in a simple invertebrate nervous system. These principles may however be relevant to all systems generating synchronized bursting activity.

As we report here, cultured neurons were seen to be spontaneously active long before the emergence of collective network behavior. Throughout the network's developmental process, prominent changes in the temporal patterns of neuronal electrical activity were apparent. These paralleled the development of the network morphology that shows distinct phases in its spontaneous organization (Shefi et al., 2002a, 2005): first, sporadic firing of single neurons as independent units changed into rhythmic burst firing in the elaborate interconnected network; next, a gradual increase in interburst interval variation and interneuronal synchronization accompanied the formation of long range connection between neuronal clusters. The small-world characteristics of mature network morphology (Shefi et al., 2002b) were reflected in the scale-free behavior of network output (Sporns et al., 2004; Eguíluz et al., 2005).

To quantify the changes in the recorded activity requires a special approach applicable both to the sequences of action potentials in the early stage and to the sequence of bursts in the later stage. The presented method of analysis enabled us to capture local and global temporal features of the activity patterns and to provide quantitative values for the descriptive observations. We were able to quantify network dynamics and decipher several new phenomena: (1) The activity of the independent neurons has relatively high regularity with low structural complexity and low functional complexity; (2) The sequences of bursting events of the mature networks reveal similar regularity to that of the individual neurons but combined with much higher structural and functional complexity; (3) During the transition periods between two types of network behaviours, the networks undergo a period of lower regularity while the complexity increases; (4) The distinct dynamics of change seen early when bursts first appear (and neurons first connect) are mirrored at the later stages of network reorganization. (5) The observed relatively high level of regularity is on the ordered side of the regularity range but close to the disorder-order boundary.

The fact that both at the early and late stages, when networks connectivity level are low and sufficiently high, the levels of regularity are similar might indicate an innate feedback mechanism to maintain the activity at a special, preferred, narrow operating range of regularity. From a dynamic systems perspective, this operating range affords the individual neuron as a functional independent unit or autonomous agents, as well as the neuronal cluster after its formation and the networks as a whole, both flexibility and stability. This was shown to be a fundamental characteristic in various studies of neuronal circuits dynamics (LinkenkaerHansen et al., 2001; Ayali et al., 2004; Bertschinger and Natschlager, 2004; Hulata et al., 2004; Haldeman and Beggs, 2005) giving the nervous system the freedom to act locally while retaining global coordination.

In an ongoing study (Shein et al., unpublished observations), we are utilizing a similar quantitative approach to that described here in the study of mammalian cortical neurons in culture aimed at supporting the generality of our findings. Our previous findings indicated that spontaneous activity of mature cortical networks in vitro show high structural and functional complexity together with relatively high regularity (Segev et al., 2002). Much work is still needed to establish the idea that evolvement of activity in our (human) nervous system undergoes similar stages of organization as those described for insect neurons. A faster connectivity scheme due to higher neuronal density may result in faster maturation of activity patterns. Recent reports suggest a strong dependence of activity development on various external conditions (Wagenaar et al., 2006b). These networks differ in their density and connectivity schemes thereby allowing us, among other things, to test this point.

In this respect, we emphasize that the choice of locust neurons as our model system provides a unique opportunity to study development of networks with special architecture-networks that are composed of linked subnetworks (neuronal clusters). This modular structure is also characteristic of real task-performing networks in the brain (Sporns et al., 2000). In accordance with the report of Sporns et al. (2000) regarding the interplay between specific topological organization and information processing in linear models, the inhomogeneous spread of neuronal connections in dense clusters was shown here to support high information capacities of neuronal activity. This was demonstrated both in our experimental and simulation results, and was not observed in model neuronal networks connected according to nearest neighbour connectivity rules. The process of increasing complexity we describe during network ontogeny may hint or reflect the process of the evolution of a centralized nervous system, during which linked ganglia fused into a network composed of overlapping subnetworks leading to greatly elevated functional complexity. 
We thank Dr. Stefano Boccaletti for stimulating discussions throughout this project, Mark Shein for inspiring conversations regarding the evolution of complexity in mammalian neural networks, and two anonymous reviewers for their constructively critical comments that have helped to improve this article.

\section{REFERENCES}

Ayali A, Fuchs E, Ben-Jacob E, Cohen A. The function of intersegmental connections in determining temporal characteristics of the spinal cord rhythmic output. Neuroscience 147:236-246.

Ayali A, Fuchs E, Zilberstein Y, Robinson A, Shefi O, Hulata E, Baruchi I, et al. 2004. Contextual regularity and complexity of neuronal activity: From stand-alone cultures to task-performing animals. Complexity 9:25-32.

Ayali A, Shefi O, Ben-Jacob E. 2002. Self-organization of two-dimensional insect neural networks. In: Gluckmam BJ, Kurths J, Pecora LM, Spano ML, Boccaletti S, editors. Experimental Chaos 2001. New York: Melville, pp 465-475.

Baruchi I, Ben Jacob E. 2007. Towards neuro-memorychip: Imprinting multiple memories in cultured neural networks. Phys Rev E 75: 050901(R).

Ben-Ari Y. 2001. Developing networks play a similar melody. Trends Neurosci 24:353-360.

Ben Jacob E, Levine H. 2001. The artistry of nature. Nature 409:985-986.

Bertschinger N, Natschlager T. 2004. Real-time computation at the edge of chaos in recurrent neural networks. Neural Comput 16:1413-1436.

Buzsaki G, Draguhn A. 2004. Neuronal oscillations in cortical networks. Science 304:1926-1929.

Chiappalone M, Bove M, Vato A, Tedesco M, Martinoia S. 2006. Dissociated cortical networks show spontaneously correlated activity patterns during in vitro development. Brain Res 6:41-53.

Cline HT. 2001. Dendritic arbor development and synaptogenesis. Curr Opin Neurobiol 11:118-126.

Corner MA, Van Pelt J, Wolters PS, Baker RE, Nuytinck R. 2002. Physiological effects of sustained blockade of excitatory synaptic transmission on spontaneously active developing neuronal networks-An inquiry into the reciprocal linkage between intrinsic biorhythms and neuroplasticity in early ontogeny. Neurosci Biobehav Rev 26:127-185.

Eguíluz VM, Chialvo DR, Cecchi GA, Baliki M, Apkarian AV. 2005. Scale-free brain functional networks. Phys Rev Lett 94:018102.

Feller MB. 1999. Spontaneous correlated activity in developing neural circuits. Neuron 22:653-656.

Fuchs E, Hulata E, Ben Jacob E, Ayali A. 2006. Adult, gender-specific behavior characterized by elevated neuronal functional complexity. Neuroreport 17:1153-1158.

Habets AMMC, Van Dongen AMJ, Van Huizen F, Corner MA. 1987. Spontaneous neuronal firing patterns in fetal rat cortical networks during development in vitro: A quantitative analysis. Exp Brain Res 69:43-52.

Haldeman C, Beggs JM. 2005. Critical branching captures activity in living neural networks and maximizes the number of metastable states. Phys Rev Lett 94:058101.

Hua JY, Smith SJ. 2004. Neural activity and the dynamics of central nervous system development. Nat Neurosci 7:327-332.

Hulata E, Baruchi I, Segev R, Shapira Y, Ben-Jacob E. 2004. Self-regulated complexity in cultured neuronal networks. Phys Rev Lett 92:198105.

Hulata E, Segev R, Shapira Y, Benveniste M, Ben-Jacob E. 2000. Detection and sorting of neural spikes using wavelet packets. Phys Rev Lett 85:4637-4640.

Hulata E, Volman V, Ben Jacob E. 2005. Self-regulated complexity in neural networks. Nat Comput 4:363386.

Kamioka H, Maeda E, Jimbo Y, Robinson HPC, Kawana A. 1996. Spontaneous periodic synchronized bursting during formation of mature patterns of connections in cortical cultures. Neurosci Lett 206:109-112.

Katz LC, Shatz CJ. 1996. Synaptic activity and the construction of cortical circuits. Science 274:1133-1138.

Linkenkaer-Hansen K, Nikouline VV, Palva JM, Ilmoniemi RJ. 2001. Long-range temporal correlations and scaling behavior in human brain oscillations. J Neurosci 21: 1370-1377.

Maeda E, Robinson HPC, Kawana A. 1995. The mechanisms of generation and propagation of synchronized bursting in developing networks of cortical neurons. J Neurosci 15:6834-6845.

Marder E, Rehm KJ. 2005. Development of central pattern generating circuits. Curr Opin Neurobiol 15:86-93.

Marom S, Shahaf G. 2002. Development, learning and memory in large random networks of cortical neurons: Lessons beyond anatomy. Q Rev Biophys 35: 63-87.

Morris C, Lecar H. 1981. Voltage oscillations in the barnacle giant muscle fiber. Biophys J 35:193-213.

O’Donovan MJ. 1999. The origin of spontaneous activity in developing networks of the vertebrate nervous system. Curr Opin Neurobiol 9:94-104.

O'Donovan MJ, Chub N. 1997. Population behavior and self-organization in the genesis of spontaneous rhythmic activity. Semin Cell Dev Biol 8:21-28.

Opitz T, De Lima AD, Voigt T. 2002. Spontaneous development of synchronous oscillatory activity during maturation of cortical networks in vitro. J Neurophysiol 88: 2196-2206.

Potter SM. 2001. Distributed processing in cultured neuronal networks. In: Nicolelis MAL, editor. Progress in Brain Research, Vol. 130. Amsterdam: Elsevier Science, pp 49-62.

Raichman N, Volman V, Ben-Jacob E. 2006. Collective plasticity and individual stability in cultured neuronal networks. Neurocomputing 69:1150-1154.

Segev R, Baruchi I, Hulata E, Ben-Jacob E. 2004. Hidden neuronal correlations in cultured networks. Phys Rev Lett 92:118102. 
Segev R, Benveniste M, Hulata E, Cohen N, Palevski A, Kapon E, Shapira Y, et al. 2002. Long term behavior of lithographically prepared in vitro neuronal networks. Phys Rev Lett 88:118102.

Shefi O, Ben Jacob E, Ayali A. 2002a. Growth morphology of two-dimensional insect neural networks. Neurocomputing 44:635-643.

Shefi O, Golding I, Segev R, Ben-Jacob E, Ayali A. 2002b. Morphological characterization of in vitro neuronal networks. Phys Rev E 66:021905.

Shefi O, Golobovitz S, Ben Jacob E, Ayali A. 2005. A twophase growth strategy in cultured neuronal networks as reflected by the distribution of neurite branching angles. J Neurobiol 62:361-368.

Sporns O, Chialvo DR, Kaiser M, Hilgetag CC. 2004. Organization, development and function of complex brain networks. Trends Cogn Sci 8:418-425.

Sporns O, Tononi G, Edelman GM. 2000. Theoretical neuroanatomy: Relating anatomical and functional connectivity in graphs and cortical connection matrices. Cereb Cortex 10:127-141.

Streit J, Tscherter A, Heuschkel MO, Renaud P. 2001. The generation of rhythmic activity in dissociated cultures of rat spinal cord. Eur J Neurosci 14:191-202.

Tateno T, Kawana A, Jimbo Y. 2002. Analytical characterization of spontaneous firing in networks of developing rat cultured cortical neurons. Phys Rev E 65: 051924.
Thiele C, Villemoes L. 1996. A fast algorithm for adapted walsh bases. Appl Comp Harm Anal 3:91-99.

Tsodyks M, Uziel A, Markram H. 2000. Synchrony generation in recurrent networks with frequency-dependent synapses. J Neurosci 20:RC50.

Van Pelt J, Corner MA, Wolters PS, Rutten WLC, Ramakers GJA. 2004. Longterm stability and developmental changes in spontaneous network burst firing patterns in dissociated rat cerebral cortex cell cultures on multielectrode arrays. Neurosci Lett 361:86-89.

Volman V, Baruchi I, Persi E, Ben-Jacob E. 2004. Generative modelling of regulated activity in cultured neuronal networks. Phys A 335:249-278.

Wagenaar DA, Nadasdy Z, Potter SM. 2006a. Persistent dynamic attractors in activity patterns of cultured neuronal networks. Phys Rev E 73:051907.

Wagenaar DA, Pine J, Potter S. 2006b. An extremely rich repertoire of bursting patterns during the development of cortical cultures. BMC Neurosci 7: 11.

Wong ROL, Ghosh A. 2002. Activity-dependent regulation of dendritic growth and patterning. Nat Rev Neurosci 3:803-812.

Zhang LI, Poo M. 2001. Electrical activity and development of neural circuits. Nat Neurosci 4:12071214. 